\title{
Uptake, Translocation, and Biotransformation of Organophosphorus Esters in Wheat (Triticum aestivum L.)
}

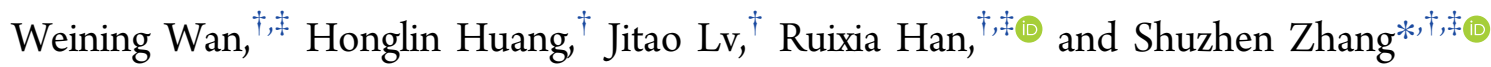 \\ ${ }^{\dagger}$ State Key Laboratory of Environmental Chemistry and Ecotoxicology, Research Center for Eco-Environmental Sciences, Chinese \\ Academy of Sciences, P.O. Box 2871, Beijing 100085, China \\ ${ }^{*}$ University of Chinese Academy of Sciences, Beijing 100049, China
}

Supporting Information

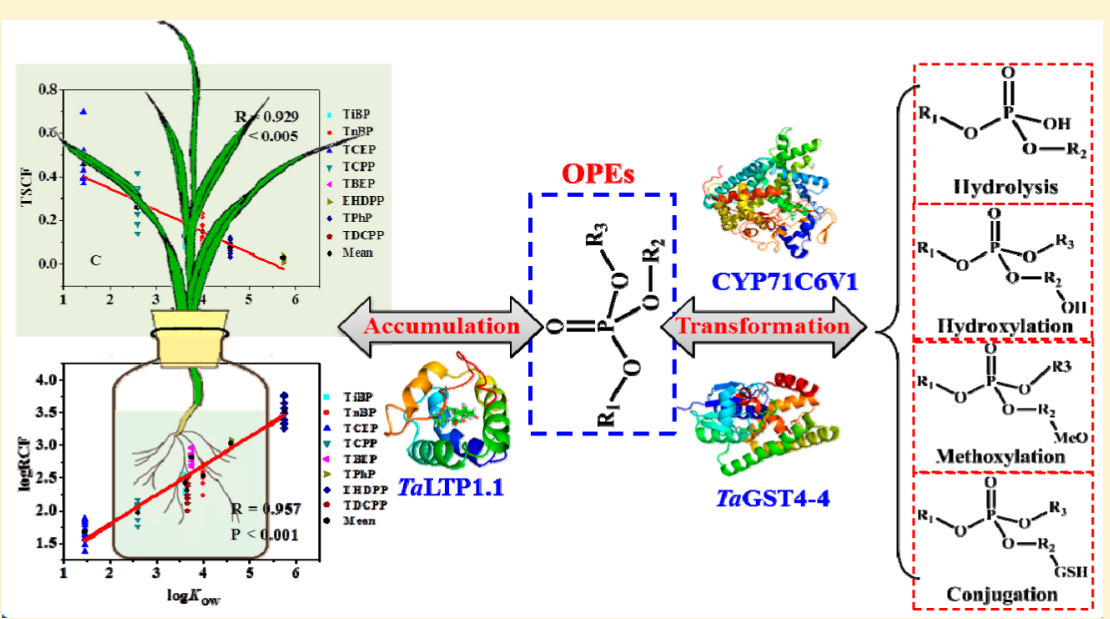

ABSTRACT: The uptake, translocation and biotransformation of organophosphate esters (OPEs) by wheat (Triticum aestivum L.) were investigated by a hydroponic experiment. The results demonstrated that OPEs with higher hydrophobicity were more easily taken up by roots, and OPEs with lower hydrophobicity were more liable to be translocated acropetally. A total of 43 metabolites including dealkylated, oxidatively dechlorinated, hydroxylated, methoxylated, and glutathione-, and glucuronideconjugated products were detected derived from eight OPEs, with diesters formed by direct dealkylation from the parent triesters as the major products, followed with hydroxylated triesters. Molecular interactions of OPEs with plant biomacromolecules were further characterized by homology modeling combined with molecular docking. OPEs with higher hydrophobicity were more liable to bind with TaLTP1.1, the most important wheat nonspecific lipid transfer protein, consistent with the experimental observation that OPEs with higher hydrophobicity were more easily taken up by wheat roots. Characterization of molecular interactions between OPEs and wheat enzymes suggested that OPEs were selectively bound to TaGST4-4 and CYP71C6v1 with different binding affinities, which determined their abilities to be metabolized and form metabolite products in wheat. This study provides both experimental and theoretical evidence for the uptake, accumulation and biotransformation of OPEs in plants.

\section{INTRODUCTION}

Except the use as insecticides in history, organophosphate esters (OPEs) are widely used as flame retardants and plasticizers in a variety of industrial applications and household products, particularly after the implementation of regulations phasing out the production and usage of polybrominated diphenyl ethers in many countries in recent years. ${ }^{1}$ OPEs are mainly used as nonreactive additives in these products and are liable to leach out to the environment. Among the OPEs, tris(1chloro-2-propyl) phosphate (TCPP), tri(1,3-dichloro-2-propyl) phosphate (TDCPP) and tri(2-butoxyethyl) phosphate (TBEP) have been suspected to be carcinogenic, ${ }^{2-4}$ whereas triethyl-chloro-phosphate (TCEP), tributyl phosphate (TnBP) and triphenyl phosphate (TPhP) have been observed to have neurotoxic effects, ${ }^{5,6}$ which may pose threats to human health.
Because of their increasing consumption volumes and potentially adverse effects, the behaviors of OPE plasticizers and flame retardants in the environment have attracted increasing attention from both the public and researchers. ${ }^{2}$ Due to the particular purpose of this study, we will use the term OPEs exclusively for OPE flame retardants and plasticizers, and not include organophosphate pesticides.

Contamination by OPEs has been detected in various environmental matrices such as soil, ${ }^{7,8}$ water, ${ }^{9,10}$ and air, ${ }^{11,12}$ and soil is one of their major sink in the environment. ${ }^{1,13,14}$

Received: April 5, 2017

Revised: October 31, 2017

Accepted: November 10, 2017

Published: November 10, 2017 
OPEs have been detected in soils, and high concentrations were found in soils at sites near contamination sources. ${ }^{7,8}$ For example, triphenyl phosphate (TPhP) was detected at concentrations of 11-3300 ng/g dry weight (dw) in the surface soils around an e-waste recycling workshop in northern Vietnam. ${ }^{7}$ In our recent work in North China, we detected eight OPEs in the soils not only at plastic waste treatment sites, with total concentrations of $38-1250 \mathrm{ng} / \mathrm{g} \mathrm{dw}$, but also in the nearby farmlands, with the total concentration range of 38-162 $\mathrm{ng} / \mathrm{g} \mathrm{dw} .{ }^{8}$ The uptake and accumulation of OPEs by crops were also evidenced in this field investigation, with all the eight OPEs detected in wheat tissues. Therefore, plant uptake and accumulation may provide the potential for OPEs to transfer into the food chain and cause a threat to human health. However, to date, studies on plant uptake of OPE plasticizers and flame retardants are scarce. There have been only two works demonstrating the uptake and translocation of OPEs by plants. ${ }^{15-17}$ Nevertheless, both of these works involved very limited OPE congeners. The factors that determine plant uptake, translocation and accumulation are far from clear.

Transformation of organic contaminants in the environment may bring additional adverse influences to bear on the environment and human health due to the fact that the biological effects of metabolites may be different compared with precursor compounds. Therefore, transformation of organic contaminants in biota and the environment has attracted increasing research attention. Several studies have detected the metabolites of OPEs in human urine samples, with diesters as the major degradation products from the parent triesters, ${ }^{18,19}$ and Reemtsma et al. ${ }^{20}$ have also found monoesters generated by further hydrolysis of diesters. So far only a few experiments have been performed on in vitro or in vivo biotransformation of OPEs. ${ }^{21-25}$ Wang et al. ${ }^{21}$ observed metabolism of TPhP by zebrafish, with diphenyl phosphate (DPhP) as the major product in the liver and intestine. Bis(2-chloroethyl) phosphate (BCEP) and bis(1-chloro-2-propyl) phosphate (BCPP) were recently reported as important metabolites of TCEP and TCPP in human liver microsomes and S9 fractions. ${ }^{22,23}$ However, these studies mainly focused on humans or animals. Biotransformation of OPE plasticizers and flame retardants in plants has not yet been reported.

OPEs all contain the same phosphate base unit. The chemical and physical properties vary extensively depending on the specific moiety of each OPE. Differences in the polarity and functional groups of OPEs have the key influences on their interaction with organisms. ${ }^{2,26,27}$ Therefore, it is expected that information on the interaction between plant biomacromolecules and OPEs would be very helpful in elucidating the molecular mechanisms of accumulation and transformation of OPEs in plants. Plant nonspecific lipid transfer proteins (nsLTPs) are biomacromolecules in all terrestrial plants and provide the key sites for lipid binding. ${ }^{28,29}$ They have been also reported to function in binding of hydrophobic xenobiotics in plants. $^{30,31}$ The isozymes glutathione-S-transferase (GST) and cytochrome P450 monooxygenases (CYP450) have been demonstrated to play the key roles in plant metabolization of organic pollutants. ${ }^{32-34}$ In wheat, TaLTP1.1 has been reported to be the most important nsLTP, ${ }^{16}$ and TaGST4-4 and CYP71C6v1 are isozymes confirmed to take part in the metabolism of herbicides. ${ }^{35,36}$ Homology modeling can construct three-dimensional (3D) models of unknown proteins, which combined with molecular docking has been successfully applied to characterize the interactions of some organic pollutants, such as polychlorinated biphenyls and pesticides, with isozymes. ${ }^{37-39}$ The function of enzymes can be predicted by their 3-D structures, and the enzyme active sites, binding modes and binding affinities between contaminants and enzymes can be characterized by molecular docking. This modeling is a valuable way to apply such information to clarify the molecular mechanisms of accumulation and biotransformation of OPEs in plants.

Therefore, the objectives of this study were to investigate the uptake, translocation and biotransformation of OPEs in wheat by a hydroponic experiment. Attempts were then made to clarify the molecular interactions between OPEs and wheat nsLTP and the isozymes TaGST4-4 and CYP71C6v1 using homology modeling combined with molecular docking. The theoretical results obtained were further used to explain the uptake and transformation of different OPEs in wheat in vivo. This study represents a thorough investigation on uptake and transformation of OPEs in plants.

\section{MATERIALS AND METHODS}

Chemicals and Reagents. Standards including TCEP, TCPP, TDCPP, TBEP, TnBP, TPhP, and EHDPP, and surrogate standards $\mathrm{d} 27-\mathrm{TnBP}, \mathrm{d} 15-\mathrm{TPhP}$, and d10-BDCPP were purchased from Wellington Laboratories, Inc. (Guelph, Ontario, Canada). The standard TiBP was obtained from AccuStandard (New Haven, CT). OPE metabolite standards BCEP, BCPP, BDCPP, BBEP, DnBP, DiBP, MnBP and the surrogate standard d10-DPhP were custom synthesized by Toronto Research Chemicals (Toronto, Ontario, Canada). Metabolite standards $\mathrm{MPhP}$ and $\mathrm{DPhP}$ were purchased from Tokyo Chemical Industry Development Co., Ltd. (Shanghai, China). Pentafluorobenzyl bromide (PFBBr) was purchased from Aldrich Chemical Co. Inc. (Steinheim, Germany). Purities of all the standards were higher than $97 \%$. The chemical names, abbreviations, formulas and properties of OPEs and metabolites are listed in Table S1 of the Supporting Information (SI). Solvents, including dichloromethane, $n$-hexane, ethyl acetate, methanol, acetonitrile, acetone and toluene, were of HPLC grade (Thermo fisher, MA). Deionized water (18.2 M $\Omega$ ) was prepared using a Milli-Q Advantage water purification system (US Millipore, Bedford, MA). All other chemicals used were of analytical grade (Sinopharm Chemical Reagent Co., Ltd., Beijing, China). Anhydrous sodium sulfate $\left(\mathrm{Na}_{2} \mathrm{SO}_{4}\right) \quad(60$ mesh), neutral silica gel, Florisil and alumina (100-200 mesh) were purchased from Fisher Scientific Inc. (Fair Lawn, NJ).

Plant Exposure Experiment. A hydroponic experiment was conducted to investigate the uptake, translocation and biotransformation of organophosphate esters (OPEs) by wheat (Triticum aestivum L.). Wheat seeds were obtained from the Chinese Academy of Agricultural Sciences, Beijing, China. Prior to germination, seeds of similar size were selected and surfacesterilized in $3 \%(\mathrm{v} / \mathrm{v}) \mathrm{H}_{2} \mathrm{O}_{2}$ for $15 \mathrm{~min}$, followed by thorough washing with sterilized deionized water, and subsequently germinated on moist filter paper in the dark at $25{ }^{\circ} \mathrm{C}$. After germination, seedlings of uniform size were transferred to glass containers containing autoclaved half-strength Hoagland nutrient solution for cultivation in a controlled environment growth chamber for 5 days. All the containers, paper and deionized water were autoclaved to prevent any possible introduction of foreign microorganisms.

Batches of 15 uniformly sized wheat seedlings were transferred to autoclaved $150 \mathrm{~mL}$ glass-stoppered flasks for exposure. The mouth of the containers was covered with 
aluminum foil and sealed with parafilm. The containers were wrapped with light-proof paper to prevent the photolysis of OPEs. Standard solutions of OPEs dissolved in methanol were added to the sterile nutrient solution to prepare the exposure solutions at nominal concentration of $75 \mu \mathrm{g} / \mathrm{L}$, which exceeded their environmentally relevant concentration, but was necessary for the determination of their accumulation and particularly their metabolism in plant tissues. The initial concentrations of TCEP, TCPP, TDCPP, TBEP, TnBP, TiBP, TPhP, and EHDPP determined in the spiked exposure solutions were 76.2 $\pm 1.5,72.5 \pm 2.6,73.8 \pm 2.2,71.4 \pm 2.4,69.6 \pm 1.8,74.0 \pm 3.1$, $75.8 \pm 2.7$, and $71.0 \pm 2.3 \mu \mathrm{g} / \mathrm{L}(n=3)$, respectively. The volume of methanol in the exposure solutions was less than $1 \%$ ( $(\mathrm{v} / \mathrm{v})$, and the $\mathrm{pH}$ was adjusted to 6.5 . Then $120 \mathrm{~mL}$ of the exposure solutions of each OPE were added individually to each of the containers. Pots were kept in a controlled environment growth chamber at a light intensity of 250 $\mu \mathrm{mol} / \mathrm{m}^{-2} \mathrm{~s}^{-1}$ provided by supplementary illumination with a photoperiod of $14 \mathrm{~h}$ each day. The day/night temperature regime was $22 / 20{ }^{\circ} \mathrm{C}$ and the relative humidity was $70 \%$. Approximately $20 \mathrm{~mL} / \mathrm{d}$ of sterile half-strength Hoagland nutrient solution saturated with oxygen was injected into each container to compensate for transpiration losses. The containers were positioned randomly and rerandomized every day. Plants were harvested at the intervals of $12,24,36,48,72,96$, 144, 192, and $240 \mathrm{~h}$. At the end of the exposure, unplanted, untreated and root exudate controls were also sampled. All the treatments were performed in triplicate.

Three types of controls were included. An untreated plant control without OPEs in the nutrient solution was used to monitor possible cross-contamination due to volatilization of OPEs. OPE-spiked nutrient solution controls without plants were set as unplanted controls to monitor any possible volatilization or abiotic degradation of OPEs. Root exudate controls were carried out mainly to explore the combined roles of root exudates and microbes, with details supplied in SI.

Sample Preparation. Roots were thoroughly rinsed with sterilized deionized water, and the rinsewater was collected and combined with the exposure solutions for the analysis of OPEs and their metabolites in solutions. Wheat seedlings were separated into roots and shoots for the subsequent analysis. All the harvested plant samples were freeze-dried at $-50{ }^{\circ} \mathrm{C}$ for 48 $\mathrm{h}$ in a lyophilizer (FD-1, Boyikang Instrument Ltd., Beijing, China), weighed and finely chopped, then stored at $-20{ }^{\circ} \mathrm{C}$ for analysis. The solution samples were extracted immediately after sampling. The extraction, cleanup, and analysis procedures were based on our previous method. ${ }^{8}$ Briefly, $0.3-0.8 \mathrm{~g}$ (dry weight) of plant samples or $50 \mathrm{~mL}$ of exposed solutions were spiked with $10 \mathrm{ng}$ of surrogates $\mathrm{d} 27-\mathrm{TnBP}$ and $\mathrm{d} 15-\mathrm{TPhP}$ for OPEs, and d10-BDCPP and d10-DPhP for metabolites. Then the plant samples were subjected to Soxhlet extraction with a $n$ hexane/DCM mixture $[1: 1(\mathrm{v} / \mathrm{v})]$, and solution samples were extracted with Oasis HLB SPE cartridges (Waters, Milford, MA). Details of extraction and cleanup of plant and solution samples are provided in the SI.

Instrumental Analysis. OPEs were separated with a HP$5 \mathrm{MS}$ column $(30 \mathrm{~m} \times 0.25 \mathrm{~mm} \times 0.25 \mu \mathrm{m})$ (Agilent $\mathrm{J} \& \mathrm{~W}$ Scientific) and analyzed by an Agilent 7890 chromatographymass spectrometry system (5975 Inert) (Agilent, Palo Alto). The MS system was operated in selected ion monitoring (SIM) mode under EI ionization for quantitation. Helium was used as the carrier gas at a flow rate of $1.5 \mathrm{~mL} / \mathrm{min}$. The oven temperature program was as follows: $1 \mathrm{~min}$ at $50{ }^{\circ} \mathrm{C}$, first ramp at $10{ }^{\circ} \mathrm{C} / \mathrm{min}$ to $200{ }^{\circ} \mathrm{C}$ ( 1 min hold), second ramp at $3{ }^{\circ} \mathrm{C} /$ min to $260{ }^{\circ} \mathrm{C}$ ( 5 min hold). The injected volume was $1 \mu \mathrm{L}$, and the injection-port temperature was $280{ }^{\circ} \mathrm{C}$ in the splitless mode. The electron energy was set at $70 \mathrm{eV}$, and the ion source temperature was $230{ }^{\circ} \mathrm{C}$.

Quantification of the metabolites including BCEP, BCPP, BDCPP, BBEP, DiBP, DnBP, DPhP, MnBP, and MPhP was performed by GC-MS (Agilent J\&W Scientific) under the SIM mode with a DB-35MS column $(60 \mathrm{~m} \times 0.25 \mathrm{~mm} \times 0.25 \mu \mathrm{m})$. The oven temperature program was as follows: $1 \mathrm{~min}$ at $70^{\circ} \mathrm{C}$, first ramp at $5{ }^{\circ} \mathrm{C} / \mathrm{min}$ to $110^{\circ} \mathrm{C}(3 \mathrm{~min}$ hold $)$, second ramp at $10{ }^{\circ} \mathrm{C} / \mathrm{min}$ to $290{ }^{\circ} \mathrm{C}(3 \mathrm{~min}$ hold $)$. Other instrument conditions were the same as the ones for the parent compounds. Possible metabolites of OPEs having no corresponding commercial standards were identified by UPLC coupled with electrospray ionization quadrupole timeof-flight tandem mass spectrometry (ESI-Q-TOF-MS) (Agilent Technologies, Santa Clara, CA). Chromatographic separation of the metabolites was achieved using a $\mathrm{BEH} \mathrm{C}_{18}$ column $(50$ $\mathrm{mm} \times 2.1 \mathrm{~mm} \times 1.7 \mu \mathrm{m}$, Waters) with $5 \mathrm{mM}$ ammonium acetate and methanol as mobile phases. The flow rate was 0.15 $\mathrm{mL} / \mathrm{min}$ and the injection volume was $20 \mu \mathrm{L}$. The products observed were assigned according to their $[\mathrm{M}-\mathrm{H}]^{-} \mathrm{m} / \mathrm{z}$ and retention time. It was assumed that the response factors for the isomers of metabolites were equal to their corresponding parent compounds based on several studies, ${ }^{40,41}$ and concentrations were semiquantified by using peak areas. Details of the analysis of OPEs and metabolites are provided in the SI, and the mass spectrometer operational parameters are available in SI Table S2.

Quality Assurance and Quality Control. Quality control was performed by regular analysis of the procedural blanks, blind duplicate samples, and random injection of solvent blanks and standards. Recoveries were obtained by the addition of 20.0, 100.0, and 500.0 ng of OPE standards, surrogate standards and metabolites in methanol at concentration of $1 \mathrm{mg} / \mathrm{mL}$ individually to the freeze-dried and pulverized untreated control plant samples and nutrient solutions before extraction and analysis. The average recoveries $(n=3)$ of different OPEs ranged from $75.6 \%$ to $90.0 \%$ for plant samples, and from $80.2 \%$ to $104.5 \%$ for solution samples, respectively. The average recoveries $(n=3)$ of the metabolites in plant and solution samples were $71.3 \%-82.4 \%$ and $77.9 \%-92.1 \%$, respectively. Recoveries of surrogate standards were as follows: $77.4-86.5 \%$ for d27-TnBP, 79.7-89.6\% for d15-TPhP, 76.2-85.7\% for $\mathrm{d} 10$ BDCPP, and $73.5-82.8 \%$ for d10-DPhP, respectively (SI Table S3). The limits of detection (LOD) for OPEs and metabolites, defined as a signal-to-noise ratio $(\mathrm{S} / \mathrm{N})$ of 3 , were in the range of $0.06-0.35 \mathrm{ng} / \mathrm{g}$ for plant tissues and $1.2-75 \mathrm{ng} / \mathrm{L}$ for solutions, respectively. Details of quality assurance and quality control are provided in SI.

Enzyme and Gene Expression Assays. Wheat roots were carefully rinsed with sterilized deionized water and finely chopped. Approximately $1 \mathrm{~g}$ of fresh root sample was used for enzyme assays. The protein level was measured by the method of Bradford ${ }^{42}$ using bovine serum albumin as the standard. GST activity was measured using the method of Habig et al., ${ }^{43}$ and CYP450 activity was assayed with the Plant CYP450 ELISA Kit (Dongge Biotechnology Co. Ltd., Beijing, China). All the enzyme activities were determined spectrophotometrically at 25 ${ }^{\circ} \mathrm{C}$ and expressed as $\mathrm{U} \cdot \mathrm{mg}^{-1}$ protein. Methods in detail are available in the SI. 

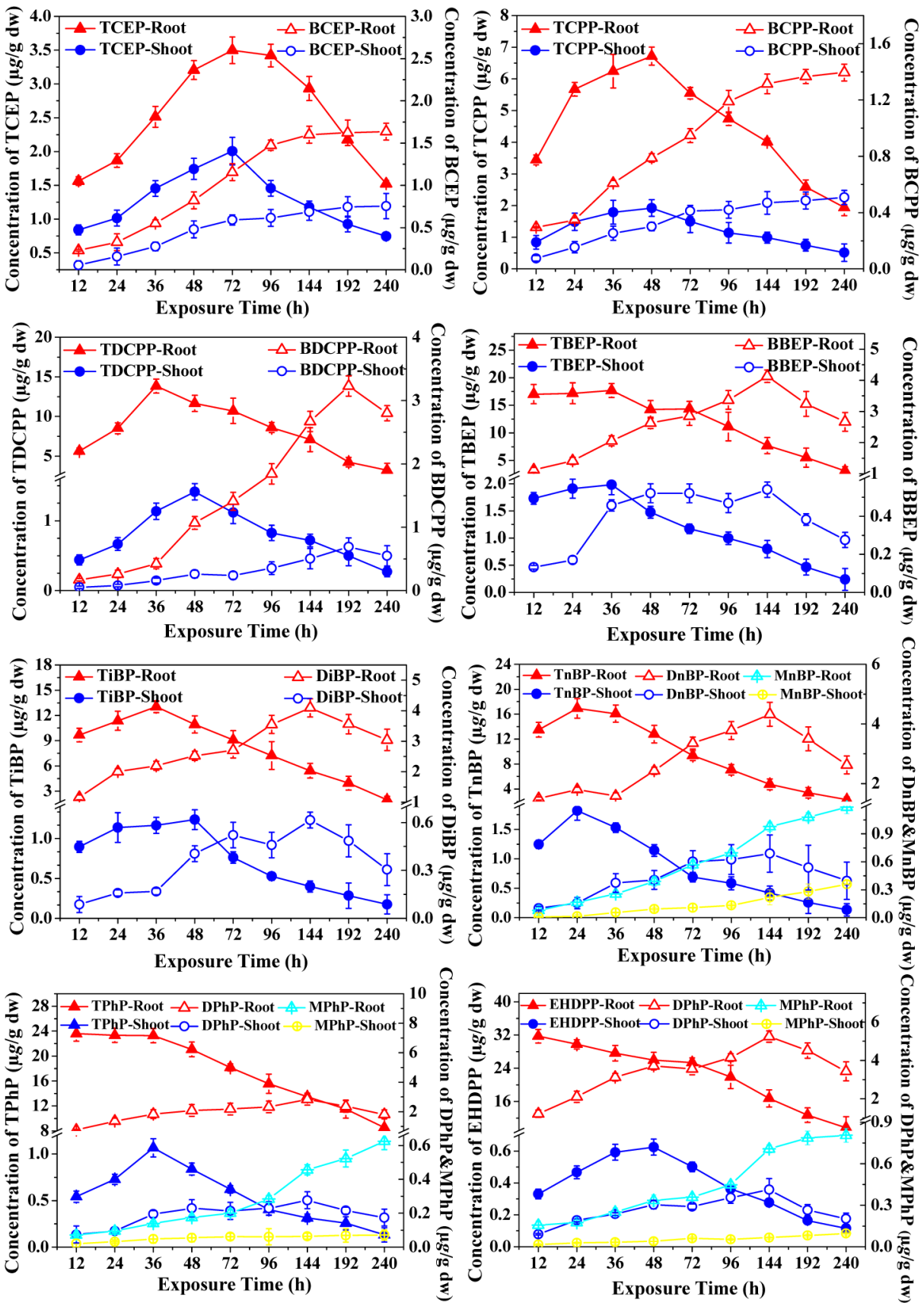

Figure 1. Time-dependent concentrations of OPEs and quantified metabolites in wheat roots and shoots. Error bars represent standard deviation values $(n=3)$.

Total RNA was isolated from frozen root samples $(100 \mathrm{mg})$, followed by precipitation with lithium chloride and then isopropanol. ${ }^{35}$ mRNA was isolated from the total RNA using a QuickPrep Micro mRNA Purification Kit (Amersham Pharmacia Biotech) as outlined in the manufacturer's directions. qRT-PCR assays were conducted in a Bio-Rad iQ5 System (Bio-Rad Company, CA). The details in procedure are provided in the SI.

Homology Modeling and Molecular Docking. TaLTP1.1, TaGST4-4 and CYP71C6v1 in wheat were chosen to investigate the interaction between OPEs and biomacromolecules. The 3-D structures of the proteins TaLTP1.1 and TaGST4-4 are available in the Protein Data Bank (PDB) with entry codes $1 \mathrm{CZ2}$ and $1 \mathrm{GWC}$, respectively. The 3-D structure of CYP71C6v1 was constructed by homology modeling. The primary sequences of CYP71C6v1 are available at the National Center for Biotechnology Information (NCBI) Web site (http://www.ncbi.nlm.nih.gov). The template protein 3PM0 (Human CYP1B1, resolution: $2.70 \AA$ ) identified by the BLAST program (http://blast.ncbi.nlm.nih.gov/Blast.cgi) was employed as the template for CYP71C6v1. The sequence identity was $25 \%$ for CYP71C6v1 to CYP1B1 (SI Figure S1A). The homology modeling and the subsequent molecular docking were based on the method of $\mathrm{Li}$ et al. ${ }^{38}$ with some modification, and the details are given in the SI. Results showed that the percentage of the residues in the core Ramachandran region was $91.8 \%$ for CYP71C6v1 (SI Figure S1B), and residues located in the unfavorable regions were far from the substrate- 

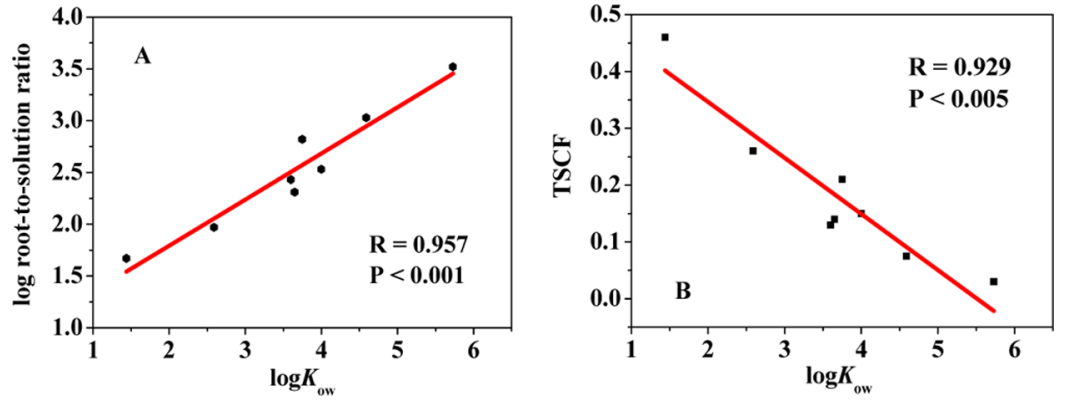

Figure 2. Relationships between the mean log root-to-solution values (A), the mean TSCF values (B) of different exposure time and log $K_{\mathrm{ow}}$ values of OPEs.

binding domain, implying negligible influence on the ligandprotein binding simulations. This suggests that the models of the plant enzymes are of reasonable quality compared with the crystal structures of the templates. TaLTP1.1 contained four $\alpha$ helices and random coils. TaGST4-4 contained nine $\alpha$ helices, four $\beta$ sheets and random coils (SI Figure S1C). CYP71C6v1 contained nine $\alpha$ helices, nine $\beta$ sheets and random coils. Molecular docking was carried out to simulate the detailed binding modes of each OPE congener to biomacromolecules using Autodock 4.2. The MOLDOCK score algorithm was employed to calculate the energy-based evaluations and to select the best orientation with the highest docking score. The lowest energy docking models were selected from the fine grid docking.

Data Analysis. Statistical analysis was performed using Microsoft Excel 2010 and Origin 8.0 (OriginLab Corporation) software. Means and standard deviations were calculated from triplicate measurements. All of the results are expressed on a dry weight basis. A $p$-value of 0.05 was taken as statistically significant.

\section{RESULTS AND DISCUSSION}

Uptake and Translocation of OPEs by Wheat Seedlings. After 10 days, $91.8-96.9 \%$ of the initial dose of OPEs in the solutions remained in the unplanted controls (SI Table S5), indicative of negligible dissipation of OPEs derived from their adsorption to the reactors, evaporation to the air and metabolization. Concentrations of OPEs in the exposure solution were time-dependent due to their plant uptake and metabolism. All the eight OPEs were detected in wheat roots. Concentrations of the OPEs in the roots increased first, reaching the peak levels at 3.50, 6.72, 13.8, 17.7, 16.9, 13.0, 23.6, and $31.7 \mu \mathrm{g} / \mathrm{g}(\mathrm{dw})$ for TCEP, TCPP, TDCPP TBEP, $\mathrm{TnBP}, \mathrm{TiBP}, \mathrm{TPhP}$, and EHDPP, respectively (Figure 1), at different exposure times, and then decreased afterward. To compare the uptake abilities of OPEs, root-to-solution ratios of OPEs were calculated for each exposure time point. Both the mean and the maximum values of log root-to-solution ratios were in the following order: EHDPP $>$ TPhP $>$ TBEP $>$ TnBP $>\mathrm{TiBP}>\mathrm{TDCPP}>\mathrm{TCPP}>\mathrm{TCEP}$. A significantly positive linear relationship was found between the maximum and the average $\log$ root-to-solution values and $\log K_{\mathrm{ow}}$ of OPEs (Figure $2 \mathrm{~A}$ and SI Figure S2A, $P<0.001$ ), demonstrating that partitioning was the major pathway for root uptake of OPEs from the exposure solutions.

The concentrations of OPEs in shoots of the blank controls after exposure are shown in SI Table S5. Only TCPP and TBEP were detected in one shoot sample at concentrations of 1.89 and $1.02 \mathrm{ng} / \mathrm{g}$, respectively, which accounted for less than $0.1 \%$ of the corresponding congeners accumulated in shoots of the exposed plants. This implied that the contribution from foliar uptake to the accumulation of OPEs in shoots was negligible under the present experimental conditions. All the OPEs were detected in shoot samples after exposures, with the maximum concentrations at $2.03,1.90,1.42,1.95,1.82,1.24,1.07$, and $0.59 \mu \mathrm{g} / \mathrm{g}(\mathrm{dw})$ for TCEP, TCPP, TDCPP, TBEP, TnBP, TiBP, TPhP and EHDPP, respectively (Figure 1), much lower than their root concentrations. Transpiration stream concentration factors (TSCFs), estimated as the ratios between the chemical concentrations in the transpiration stream and the exposure solution, have been widely used as the descriptor of acropetal translocation of organic chemicals. ${ }^{44-46}$ The TSCFs for OPEs were calculated for different exposure intervals. Both the mean and the maximum TSCF values were found to be negatively correlated with their $\log K_{\mathrm{ow}}$ values (Figure $2 \mathrm{~B}$ and SI Figure $S 2 B, P<0.005)$, suggesting that OPEs with lower hydrophobicity were more prone to be translocated from roots to shoots. In particular, the relationship between the maximum TSCF versus $\log K_{\text {ow }}$ followed a nearly sigmoidal curve (SI Table S6), similar to the one obtained by Dettenmaier et al., ${ }^{46}$ when they investigated the plant uptake of 25 organic chemicals with $\log K_{\text {ow }}$ ranging in -0.8 to 5 , similar to $\log K_{\text {ow }}$ values of the target OPEs in the present study.

Biotransformation of OPEs in Wheat Seedlings. Seven diesters and two monoesters were detected and quantified in wheat roots and shoots, with their concentrations higher in roots than in shoots (Figure 1); but none of them were found in the untreated and unplanted controls (SI Table S5). All the metabolites were detected in wheat tissues after exposure for only $12 \mathrm{~h}$, indicating metabolism occurred very rapidly. Concentrations of BDCPP, BBEP, DiBP, DnBP, and DPhP in wheat roots and shoots increased first and then decreased noticeably, while concentrations of BCEP and BCPP kept increasing. This suggests that BCEP and $\mathrm{BCPP}$ were relatively stable, whereas the other metabolites might be further metabolized inside plants. Concentrations of $\mathrm{MnBP}$ and $\mathrm{MPhP}$ in both roots and shoots kept increasing during the exposure time, being most likely attributed to the further continuous transformation of diesters to monoesters. Metabolites in wheat should derive from a combination of root uptake following metabolism of the parent OPE compounds in the exposure solutions and metabolism occurring inside plants. Although we paid particular attention to avoiding metabolism of OPEs in the exposure solutions caused by microorganisms by sterilizing the culture solution and sealing the exposure reactors, metabolites were detected in the culture solutions during the exposure time (SI Figure S3). This could have occurred because root-associated microbes can be inoculated in 

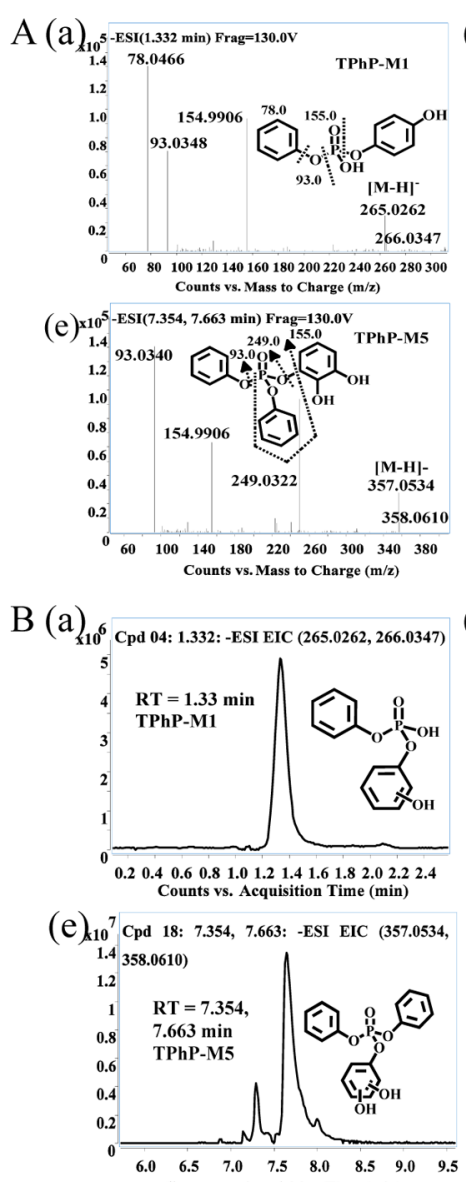

Counts vs. Acquisition Time (min) (b)
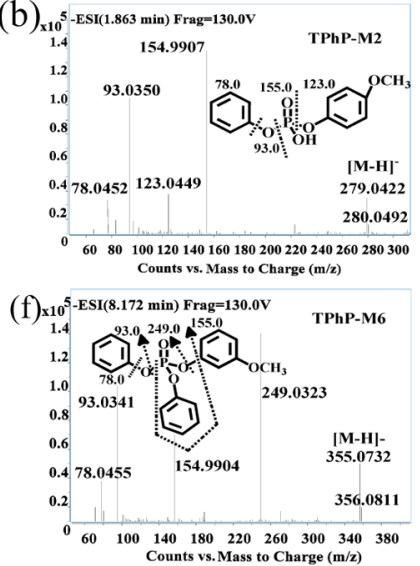

(b)
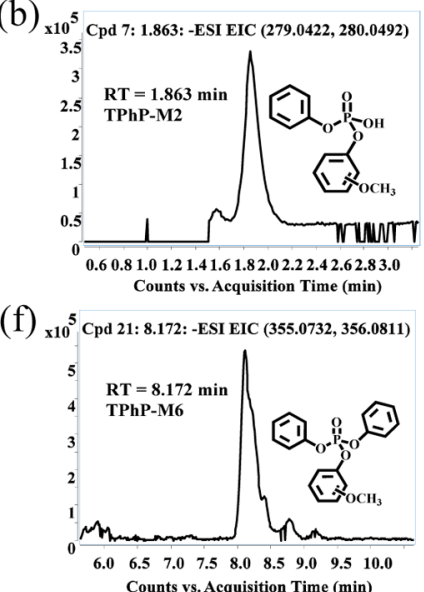

(c)

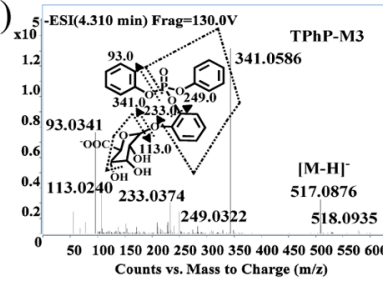

(d)

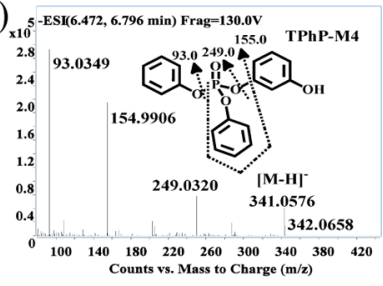

(g)

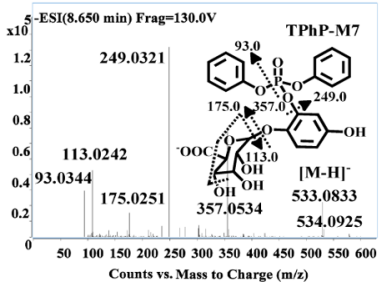

(c)

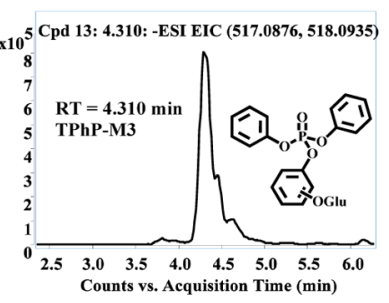

(d)

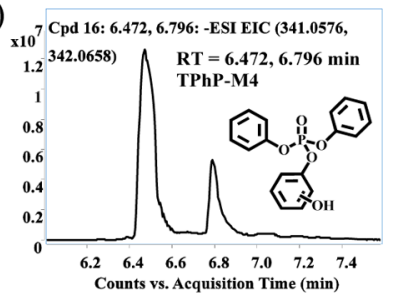

(g)

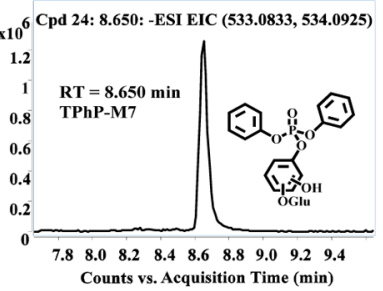

Figure 3. Identification of the potential metabolites of OPEs in wheat roots by UPLC-Q-TOF-MS taking TPhP as an example. The mass spectra (A) indicate the types of fragment ions and masses (shown as arrows and dotted lines). The chromatograms (B) provide the approximate retention times of the metabolites, which include monohydroxylated diphenyl phosphate (M1), monomethoxylated diphenyl phosphate (M2), mono- glucuronic acid conjugated metabolites after hydroxylation (M3), monohydroxylated TPHP (M4), dihydroxylated TPHP (M5), monomethoxylated TPHP (M6), and glucuronic acid conjugated metabolites after dihydroxylation (M7).

the solution during plant cultivation, ${ }^{47}$ which would result in metabolism of OPEs in the solutions to some extent. This was confirmed by our observation on the metabolism of OPEs in the fresh root-exudate solutions (see results in SI Table S7). Further, the metabolites are generally more polar than their parent compounds. Therefore, OPE metabolites should have lower $\log K_{\mathrm{ow}}$ and therefore lower $\log$ root-to-solution values than the parent OPEs if only uptake occurred. However, the log root-to-solution values of the metabolites were found to be much higher than those of the corresponding parent compounds (SI Table S8). This suggested the occurrence of metabolism of OPEs inside plants besides root uptake from exposure solutions.

In addition to the metabolites quantified above, we observed some unidentified peaks in the chromatograph (SI Figure S4), likely belonging to some other unidentified metabolites. The mass balance was tested for each exposure time by calculating the percentages of the total mass of each OPE and its metabolites in the whole plant and the exposure solution to the initial amount added (SI Table S9). It was found that the recoveries of OPEs decreased with increasing plant exposure time, and $44.3 \%$ to $83.5 \%$ of the OPEs were recovered at the end of the exposure. These results might also suggest the existence of some unidentified metabolites. Unfortunately, commercial standards of the potential metabolites are unavailable, precluding accurate quantification of their concentrations. Therefore, the putative metabolites were identified by UPLC-ESI-Q-TOF-MS, and the mass spectra and chromatograms were collected (Figure 3 ). The products observed were assigned according to their $[\mathrm{M}-\mathrm{H}]^{-} m / z$ and retention time, and the chemical information for the metabolites identified is provided in SI Table S10. A total of 34 metabolites belonging to eight OPEs respectively were identified in wheat tissues (SI Figure S5). The levels of the products were higher in roots than in shoots. TCEP exhibited the fewest kinds of metabolites while TBEP had the most. Oxidatively dechlorinated, hydroxylated, and glutathione (GSH)-conjugated products were found to be derived from the halogenated OPEs, whereas the metabolites detected for nonhalogenated OPEs included oxidative-dealkylated, hydroxylated, methoxylated and glucuronide-conjugated products (see SI Figure S6 for details). Similar oxidativedealkylation, GSH- and glucuronide-conjugated products have been reported for organophosphorus pesticides in previous works. ${ }^{48,49}$ The metabolites containing hydroxyl, especially the hydroxylated triesters, accounted for the largest amounts of the total products in both roots and shoots for all the OPEs. Su et al. $^{24,25}$ also detected mono- and di- hydroxylated TPhP isomers in a test using chicken embryonic hepatocyte screening, with their levels increasing over the exposure time, and based on this observation they highlighted the importance of the hydrox- 


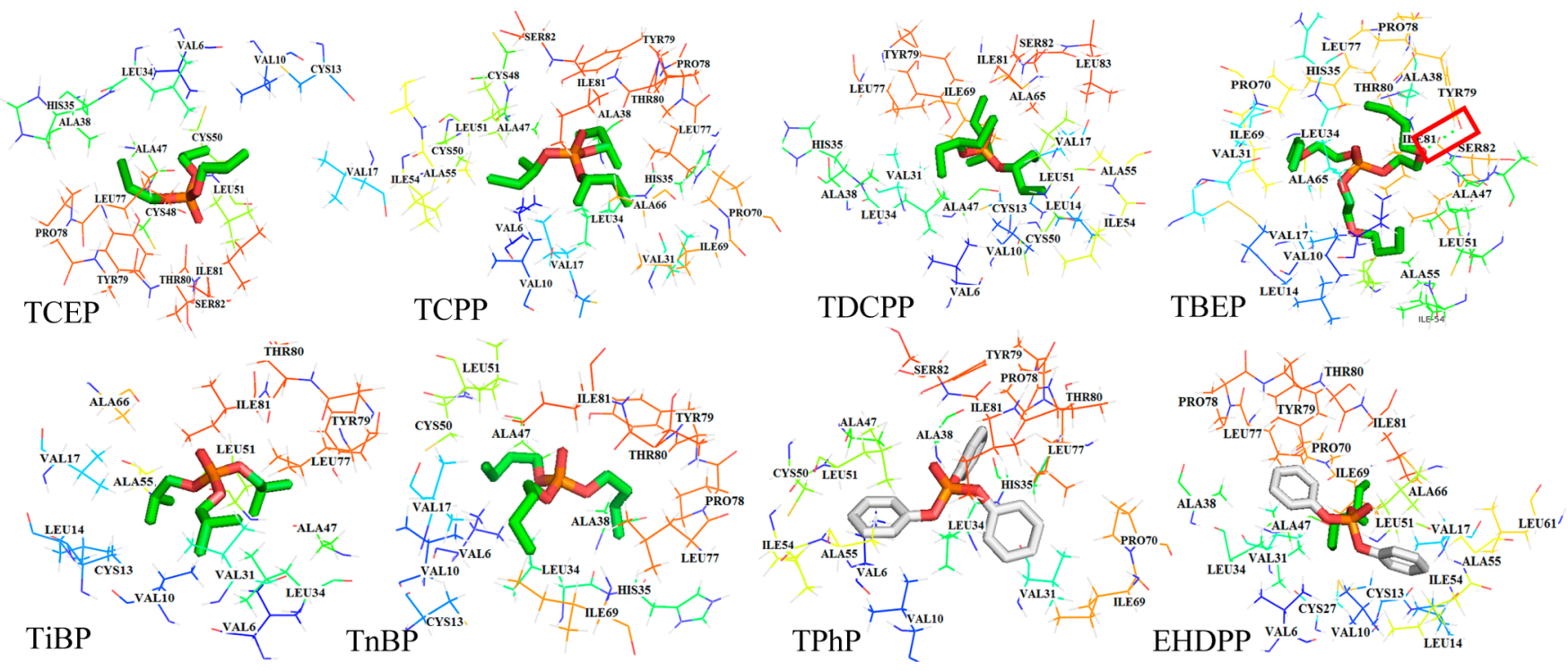

Figure 4. Residues in the active sites of TaLTP1.1 interacting with OPE congeners. The hydrogen bonds formed between TBEP and the residue Tyr79 are displayed with green dotted lines.

ylation pathway. Van den Eede et al. ${ }^{22}$ also identified a considerable contribution of hydroxylated products of TCPP, $\mathrm{TBEP}$, and TPhP to metabolism of OPEs in human liver cells. To our knowledge, this is the first time the existence of hydroxylated OPEs in plants has been reported, to which we should pay particular attention since hydroxylated metabolites are sometimes more toxic than their parent compounds. ${ }^{50-54}$ Patterns of metabolites from diverse OPE congeners were distinct. For instance, monoesters were found for all nonhalogenated OPEs but none was identified for the halogenated ones, and the GSH-conjugated products were detected as the phase II metabolites for halogenated OPEs, whereas the glucuronide-conjugated ones instead of the GSH-adducts were found for nonhalogenated OPEs. In addition, among the three halogenated OPEs, the direct hydroxylation metabolite without removing the $\mathrm{Cl}$ atom only appeared for TCPP, and the products of further methoxylation after hydroxylation were found for aryl phosphates (SI Figure S6), which had not been reported in previous research on the metabolism of TPhP. The hydroxylated and methoxylated diesters in wheat might be formed through hydroxylation and methoxylation of their corresponding diesters or through dealkylation of their hydroxylated and methoxylated triesters; which of these is the main transformation pathway needs further investigation. Mass balance was then further estimated by including both the quantified and semiquantified metabolites (SI Table S11). $71.1 \%$ to $94.6 \%$ of the initial OPEs were recovered at the end of exposure, which were improved approximately $8.5 \%$ to $30.2 \%$ compared to the data acquired with only the quantified metabolites included. Improvement was particularly achieved for TCPP, TBEP, and TPhP, which had relatively more metabolites detected.

Molecular Interactions between OPEs and Plant Biomacromolecules. The results above showed the diverse accumulation and transformation of different OPEs in wheat tissues. To obtain further insight into the mechanisms involved in these processes, molecular interactions between OPEs and plant biomacromolecules were characterized using homology modeling combined with molecular docking. Dynamic changes of GST and CYP450 activities and gene expressions in wheat roots were first investigated in order to determine their roles in the metabolization of OPEs in wheat plants. Compared with those of blank controls, the significantly increasing activities of the two enzymes $(P<0.05$, SI Figure S7) and the up-regulation in gene expressions of TaGST4-4 and CYP71C6v1 (Fold change $\geq 2$, SI Figure S7) were observed during the exposure time, demonstrating that they were involved in the metabolism of OPEs in wheat. The results provided an experimental basis for molecular docking and for the analysis of interactions between OPEs and the plant biomacromolecules. It was found that all the OPEs could be docked into the active cavities of wheat biomacromolecules TaLTP1.1, TaGST4-4, and CYP71C6v1 (Figure 4 and SI Figure S8). The main residues in the active sites including Leu34, Ala38, Ala47, Leu51, Leu77, Tyr79, and Ile81 belong to TaLTP1.1, Pro14, Ser15, Phe17, Arg114, Arg118, Trp171, and Arg222 to TaGST4-4, and Ser78, Phe79, Leu187, Ala322, Glu325, and Met506 to CYP71C6v1, respectively, were found to be located within a distance of $3 \AA$ to the OPE congeners (SI Table S12, Figure 4, and SI Figure S8), implying their important roles in binding to the OPE congeners. All these amino acid residues were hydrophobic and were probably bound with OPEs mainly via hydrophobic interactions. Furthermore, several OPEs formed hydrogen bonds with the residues, such as TBEP with the residue Tyr79 of TaLTP1.1; TCEP with Ser15, TDCPP with Arg114, and TPhP with Arg222 of TaGST4-4; and TiBP with Ser78, and TPhP with Glu325 of CYP71C6v1, respectively (Figure 4 and SI Figure S8). The interaction energies of TCEP, TCPP, TDCPP, TBEP, TnBP, TiBP, TPhP, and EHDPP were from -5.38 to $-8.10 \mathrm{kcal} / \mathrm{mol}$ for TaLTP1.1, from -4.78 to $-6.33 \mathrm{kcal} / \mathrm{mol}$ for TaGST4-4, and from -3.02 to $-6.82 \mathrm{kcal} /$ mol for CYP71C6v1, respectively (SI Table S13). Compared with the binding affinity values of the van der Waals force, the effect of the electrostatic energy was negligible (SI Table S13), suggesting that OPEs were bound to the active sites of plant biomacromolecules mainly via van der Waals forces. The binding affinities varied markedly among different OPEs. The docking energies for OPEs to TaLTP1.1 were found to have a significantly negative correlation to the $\log K_{\text {ow }}$ values of OPEs (SI Figure S9, $P<0.005$ ), that is to say, OPEs with higher 
hydrophobicity were more liable to bind with TaLTP1.1, similar to the uptake abilities of OPEs quantified and presented as $\log$ root-to-solution values. Therefore, a consistent conclusion was achieved from the experimental and molecular docking results that hydrophobic partitioning into plant biomacromolecules was the major pathway for the uptake of OPEs by wheat. Considering the interaction of OPEs with the wheat enzymes, the nonhalogenated OPEs were found to have more negative binding energies and therefore were more liable to fit into the active sites of CYP71C6v1 and TaGST4-4 compared with the halogenated OPEs, supporting the experimental observation that nonhalogenated OPEs such as TBEP, TiBP and EHDPP were more liable to be metabolized. The binding energies of halogenated OPEs for TaGST4-4 were more negative than those for CYP71C6v1, indicating that halogenated OPEs were more prone to bind with wheat GST enzyme. This is in accordance with the experimental results that GSH conjugate metabolites were found for halogenated OPEs. By contrast, the nonhalogenated OPEs were preferentially bound with CYP71C6v1 rather than TaGST4-4, in line with the experimental observation of the existence of the monoesters and the dihydroxylated products, which have been evidenced to be mainly produced by the CYP isozymes. ${ }^{55,56}$ Moreover, it was worth noting that TBEP had the most negative binding energies for both TaGST4-4 and CYP71C6v1, and therefore possessed the strongest interactions with wheat enzymes, which was in agreement with the experimental evidence that TBEP was the most readily metabolized and generated the most metabolites in wheat. Therefore, the conclusion can be made that the diverse accumulation and transformation of OPE congeners may derive from their different binding modes and affinities to plant biomacromolecules.

Environmental Implications. This study provides both experimental and theoretical evidence for the uptake, accumulation and biotransformation of OPEs in wheat plants. Uptake by roots and translocation to shoots indicate the potential for OPEs to transfer into the food chain. Transformation of OPEs in plants was evidenced with a total of 43 metabolites detected, which may cause adverse effects on the environment and human health. Molecular interaction between OPEs and the wheat biomacromolecules nsLTP, GST, and P450 explained the diverse accumulation and transformation of OPEs in wheat tissues observed in the experiments. Results obtained by this study are useful to gain further understanding of the fates of OPEs in the environment. Further research is necessary on the generation and behaviors of OPE metabolites in the soil-plant system, which is critical for evaluating the fate and potential toxicity of OPEs in the environment.

\section{ASSOCIATED CONTENT}

\section{S Supporting Information}

The Supporting Information is available free of charge on the ACS Publications website at DOI: 10.1021/acs.est.7b01758.

Additional figures and experimental details regarding extraction and analytical methods of OPE congeners and metabolites, homology modeling and molecular docking methods, OPE metabolite identification and distribution, mass balance of OPEs, 3-D structures of plant biomacromolecules, and OPEs binding with plant biomacromolecules (PDF)

\section{AUTHOR INFORMATION}

\section{Corresponding Author}

*Phone: +86-10-62849683; fax: +86-10-62923563; e-mail: szzhang@rcees.ac.cn.

ORCID $\odot$

Ruixia Han: 0000-0003-2357-8019

Shuzhen Zhang: 0000-0002-3494-9674

\section{Notes}

The authors declare no competing financial interest.

\section{ACKNOWLEDGMENTS}

This work was funded by the National Natural Science Foundation of China (Projects 21537005 and 21621064) and the Strategic Priority Research Program of the Chinese Academy of Sciences (Grant XDB14020202).

\section{REFERENCES}

(1) Wei, G. L.; Li, D. Q.; Zhuo, M. N.; Liao, Y. S.; Xie, Z. Y.; Guo, T. L.; Li, J. J.; Zhang, S. Y.; Liang, Z. Q. Organophosphorus flame retardants and plasticizers: Sources, occurrence, toxicity and human exposure. Environ. Pollut. 2015, 196, 29-46.

(2) Van der Veen, I.; de Boer, J. Phosphorus flame retardants: properties, production, environmental occurrence, toxicity and analysis. Chemosphere 2012, 88 (10), 1119-1153.

(3) World Health Organization. Flame Retardants: Tris(chloropropyl) phosphate and tris(2-chloroethyl) phosphate. Environmental Health Criteria 209; World Health Organization, Geneva, Switzerland, 1998.

(4) World Health Organization. Flame Retardants: Tris(2-butoxyethyl) phosphate, tris(2-ethylhexyl) phosphate and tetrakis(hydroxymethyl) phosphonium salts. Environmental Health Criteria 209; World Health Organization, Geneva, Switzerland, 2000a.

(5) World Health Organization. Triphenyl Phosphate. Environmental Health Criteria 111; World Health Organization, Geneva, Switzerland, 1991a.

(6) World Health Organization. Tri-n-butyl Phosphate. Environmental Health Criteria 112; World Health Organization, Geneva, Switzerland, 1991b.

(7) Matsukami, H.; Tue, N. M.; Suzuki, G.; Someya, M.; Tuyen, L. H.; Viet, P. H.; Takahashi, S.; Tanabe, S.; Takigami, H. Flame retardant emission from e-waste recycling operation in northern Vietnam: Environmental occurrence of emerging organophosphorus esters used as alternatives for PBDEs. Sci. Total Environ. 2015, 514, 492-499.

(8) Wan, W. N.; Zhang, S. Z.; Huang, H. L.; Wu, T. Occurrence and distribution of organophosphorus esters in soils and wheat plants in a plastic waste treatment area in China. Environ. Pollut. 2016, 214, 349353.

(9) Andresen, J. A.; Grundmann, A.; Bester, K. Organophosphorus flame retardants and plasticisers in surface waters. Sci. Total Environ. 2004, 332 (1-3), 155-166.

(10) Bollmann, U. E.; Möller, A.; Xie, Z. Y.; Ebinghaus, R.; Einax, J. $\mathrm{W}$. Occurrence and fate of organophosphorus flame retardants and plasticizers in coastal and marine surface waters. Water Res. 2012, 46 (2), 531-538.

(11) Castro-Jimenez, J.; Berrojalbiz, N.; Pizarro, M.; Dachs, J. Organophosphate ester (OPE) flame retardants and plasticizers in the open Mediterranean and Black Seas atmosphere. Environ. Sci. Technol. 2014, 48 (6), 3203-3209.

(12) Salamova, A.; Ma, Y. N.; Venier, M.; Hites, R. A. High levels of organophosphate flame retardants in the Great Lakes atmosphere. Environ. Sci. Technol. Lett. 2014, 46 (16), 8653-8660.

(13) Mihajlovic, I.; Fries, E. Atmospheric deposition of chlorinated organophosphate flame retardants (OFR) onto soils. Atmos. Environ. 2012, 56 (5), 177-183. 
(14) Mihajlovic, I.; Miloradov, M. V.; Fries, E. Application of Twisselmann extraction, SPME, and GC-MS to assess input sources for organophosphate esters into soil. Environ. Sci. Technol. 2011, 45 (6), 2264-2269.

(15) Eggen, T.; Heimstad, E. S.; Stuanes, A. O.; Norli, H. R. Uptake and translocation of organophosphate and other emerging contaminants in food and forage crops. Environ. Sci. Pollut. Res. 2013, 20 (7), $4520-4531$.

(16) Hyland, K. C.; Blaine, A. C.; Dickenson, E. R.; Higgins, C. P. Accumulation of contaminants of emerging concern in food cropspart 1: Edible strawberries and lettuce grown in reclaimed water. Environ. Toxicol. Chem. 2015, 34 (10), 2213-2221.

(17) Hyland, K. C.; Blaine, A. C.; Higgins, C. P. Accumulation of contaminants of emerging concern in food crops-part 2: Plant distribution. Environ. Toxicol. Chem. 2015, 34 (10), 2222-2230.

(18) Van den Eede, N.; Neels, H.; Jorens, P. G.; Covaci, A. Analysis of organophosphate flame retardant diester metabolites in human urine by liquid chromatography electrospray ionisation tandem mass spectrometry. J. Chromatogr. A 2013, 1303 (16), 48-53.

(19) Hoffman, K.; Daniels, J. L.; Stapleton, H. M. Urinary metabolites of organophosphate flame retardants and their variability in pregnant women. Environ. Int. 2013, 63C (3), 169-172.

(20) Reemtsma, T.; Lingott, J.; Roegler, S. Determination of 14 monoalkyl phosphates, dialkyl phosphates and dialkyl thiophosphates by LC-MS/MS in human urinary samples. Sci. Total Environ. 2011, 409 (10), 1990-1993.

(21) Wang, G. W.; Du, Z. K.; Chen, H. Y.; Su, Y.; Gao, S. X.; Mao, L. Tissue-specific accumulation, depuration and transformation of triphenyl phosphate (TPHP) in adult zebrafish (Danio rerio). Environ. Sci. Technol. 2016, 50 (24), 13555-13564.

(22) Van den Eede, N.; Maho, W.; Erratico, C.; Neels, H.; Covaci, A. First insights in the metabolism of phosphate flame retardants and plasticizers using human liver fractions. Toxicol. Lett. 2013, 223 (1), 9-15.

(23) Van den Eede, N.; Tomy, G.; Tao, F.; Halldorson, T.; Harrad, S.; Neels, H.; Covaci, A. Kinetics of tris (1-chloro-2-propyl) phosphate (TCIPP) metabolism in human liver microsomes and serum. Chemosphere 2016, 144, 1299-1305.

(24) Su, G. Y.; Crump, D.; Letcher, R. J.; Kennedy, S. W. Rapid in vitro metabolism of the flame retardant triphenyl phosphate and effects on cytotoxicity and mRNA expression in chicken embryonic hepatocytes. Environ. Sci. Technol. 2014, 48 (22), 13511-13519.

(25) Su, G. Y.; Letcher, R. J.; Crump, D.; Gooden, D. M.; Stapleton, $\mathrm{H}$. M. In vitro metabolism of the flame retardant triphenyl phosphate in chicken embryonic hepatocytes and the importance of the hydroxylation pathway. Environ. Sci. Technol. Lett. 2015, 2 (4), 100104.

(26) Zhao, F. R.; Wan, Y.; Zhao, H. Q.; Hu, W. X.; Mu, D.; Webster, T. F.; Hu, J. Y. Levels of blood organophosphorus flame retardants and association with changes in human sphingolipid homeostasis. Environ. Sci. Technol. 2016, 50 (16), 8896-8903.

(27) Ren, X. M.; Cao, L. Y.; Yang, Y.; Wan, B.; Wang, S. F.; Guo, L. $\mathrm{H}$. In vitro assessment of thyroid hormone receptor activity of four organophosphate esters. J. Environ. Sci. 2016, 45 (7), 185-190.

(28) Kader, J. C. Lipid-transfer proteins in plants. Annu. Rev. Plant Physiol. Plant Mol. Biol. 1996, 47 (47), 627-654.

(29) Wong, L. H.; Levine, T. P. Lipid transfer proteins do their thing anchored at membrane contact sites $\cdots$ but what is their thing? Biochem. Soc. Trans. 2016, 44 (2), 517-527.

(30) Pato, C.; Borgne, M. L.; Baut, G. L.; Pape, P. L.; Marion, D.; Douliez, J. P. Potential application of plant lipid transfer proteins for drug delivery. Biochem. Pharmacol. 2001, 62 (5), 555-560.

(31) Salminen, T. A.; Blomqvist, K.; Edqvist, J. Lipid transfer proteins: Classification, nomenclature, structure, and function. Planta 2016, 244 (5), 971-997.

(32) Amrani, A. E.; Dumas, A. S.; Wick, L. Y.; Yergeau, E.; Berthomé, R. Omics" insights into PAH degradation toward improved green remediation biotechnologies. Environ. Sci. Technol. 2015, 49 (19), 11281-11291.
(33) Huang, H. L.; Zhang, S. Z.; Wang, S.; Lv, J. T. In vitro biotransformation of PBDEs by root crude enzyme extracts: Potential role of nitrate reductase $(\mathrm{NaR})$ and glutathione S-transferase (GST) in their debromination. Chemosphere 2013, 90 (6), 1885-1892.

(34) Zhai, G.; Lehmler, H. J.; Schnoor, J. L. Inhibition of cytochromes $\mathrm{P} 450$ and the hydroxylation of 4-monochlorobiphenyl in whole poplar. Environ. Sci. Technol. 2013, 47 (13), 6829-6835.

(35) Xiang, W. S.; Wang, X. J.; Ren, T. R.; Ju, X. L. Expression of a wheat cytochrome $\mathrm{P} 450$ monooxygenase in yeast and its inhibition by glyphosate. Pest Manage. Sci. 2005, 61 (4), 402-406.

(36) Thom, R.; Cummins, I.; Dixon, D. P.; Edwards, R.; Cole, D. J.; Lapthorn, A. J. Structure of a tau class glutathione S-transferase from wheat active in herbicide detoxification. Biochemistry 2002, 41 (22), $7008-7020$

(37) Yoo, J.; Hirano, M.; Mizukawa, H.; Nomiyama, K.; Agusa, T.; Kim, E. Y.; Tanabe, S.; Iwata, H. In vitro and in silico analyses for predicting hepatic cytochrome $\mathrm{P} 450$-dependent metabolic potencies of polychlorinated biphenyls in the Baikal Seal. Environ. Sci. Technol. 2015, 49 (24), 14588-14596.

(38) Li, Q. F.; Fang, Y. P.; Li, X. X.; Zhang, H.; Liu, M. M.; Yang, H. B.; Kang, Z.; Li, Y.; Wang, Y. H. Mechanism of the plant cytochrome P450 for herbicide resistance: A modelling study. J. Enzyme Inhib. Med. Chem. 2013, 28 (6), 1182-1191.

(39) Rupasinghe, S.; Schuler, M. A. Homology modeling of plant cytochrome P450s. Phytochem. Rev. 2006, 5 (2), 473-505.

(40) Abdallah, M. A. E.; Uchea, C.; Chipman, J. K.; Harrad, S. Enantioselective biotransformation of hexabromocyclododecane by in vitro rat and trout hepatic sub-cellular fractions. Environ. Sci. Technol. 2014, 48 (5), 2732-2740.

(41) Marvin, C. H.; Macinnis, G.; Alaee, M.; Arsenault, G.; Tomy, G. T. Factors influencing enantiomeric fractions of hexabromocyclododecane measured using liquid chromatography/tandem mass spectrometry. Rapid Commun. Mass Spectrom. 2007, 21 (12), 1925-1930.

(42) Bradford, M. M. A rapid and sensitive method for quantitation of microgram quantities of protein utilizing principle of protein dyebinding. Anal. Biochem. 1976, 72 (1-2), 248-254.

(43) Habig, W. H.; Pabst, M. J.; Fleischner, G.; Gatmaitan, Z.; Arias, I. M.; Jakoby, W. B. The identity of glutathione S-transferase B with ligandin, a major binding protein of liver. Proc. Natl. Acad. Sci. U. S. A. 1974, 71 (10), 3879-3882.

(44) Briggs, G. G.; Bromilow, R. H.; Evans, A. A. Relationships between lipophilicity and root uptake and translocation of non-ionised chemicals by barley. Pestic. Sci. 1982, 13 (5), 495-504.

(45) Burken, J. G.; Schnoor, J. L. Predictive relationships for uptake of organic contaminants by hybrid poplar trees. Environ. Sci. Technol. 1998, 32 (21), 3379-3385.

(46) Dettenmaier, E. M.; Doucette, W. J.; Bugbee, B. Chemical hydrophobicity and uptake by plant roots. Environ. Sci. Technol. 2009, 43 (2), 324-329.

(47) Pangesti, N.; Pineda, A.; Pieterse, C. M. J.; Dicke, M.; Van Loon, J. J. A. Two-way plant-mediated interactions between root-associated microbes and insects: From ecology to mechanisms. Front. Plant Sci. 2013, 4 (1), 414.

(48) Casida, J. E.; Lykken, L. Metabolism of organic pesticide chemicals in higher plants. Annu. Rev. Plant Physiol. 1969, 20 (1), 607-636.

(49) Casida, J. E. Mode of action of pesticides, metabolism of organophosphorus insecticides in relation to their antiesterase activity, stability, and residual properties. J. Agric. Food Chem. 1956, 4 (9), $772-785$.

(50) Su, G. Y.; Xia, J.; Liu, H. L.; Lam, M. H. W.; Yu, H. X.; Giesy, J. P.; Zhang, X. W. Dioxin-like potency of $\mathrm{HO}$ - and $\mathrm{MeO}$-analogues of PBDEs' the potential risk through consumption of fish from Eastern China. Environ. Sci. Technol. 2012, 46 (19), 10781-10788.

(51) Xu, X. H.; Huang, H. L.; Wen, B.; Wang, S.; Zhang, S. Z. Phytotoxicity of brominated diphenyl ether-47 (BDE-47) and its hydroxylated and methoxylated analogues (6-OH-BDE-47 and 6MeO-BDE-47) to maize (Zea mays L.). Chem. Res. Toxicol. 2015, 28 (3), 510-517. 
(52) Meerts, I.; Letcher, R. J.; Hoving, S.; Marsh, G.; Bergman, A.; Lemmen, J. G.; van der Burg, B.; Brouwer, A. In vitro estrogenicity of polybrominated diphenyl ethers, hydroxylated PDBEs, and polybrominated bisphenol A compounds. Environ. Health Perspect. 2001, 109 (4), 399-407.

(53) Layton, A. C.; Sanseverino, J.; Gregory, B. W.; Easter, J. P.; Sayler, G. S.; Schultz, T. W. In vitro estrogen receptor binding of PCBs: Measured activity and detection of hydroxylated metabolites in a recombinant yeast assay. Toxicol. Appl. Pharmacol. 2002, 180 (3), 157-163.

(54) Kojima, H.; Takeuchi, S.; Van den Eede, N.; Covaci, A. Effects of primary metabolites of organophosphate flame retardants on transcriptional activity via human nuclear receptors. Toxicol. Lett. 2016, 245, 31-39.

(55) Hoagland, R. E.; Zablotowicz, R. M.; Hall, J. C. Pesticide metabolism in plants and microorganisms. Weed Sci. 2003, 51 (4), 472-495.

(56) Siminszky, B. Plant cytochrome P450-mediated herbicide metabolism. Phytochem. Rev. 2006, 5 (2), 445-458. 